\title{
Editorial:
}

\section{The Necessity and Challenges of Clinical Research Involving Children ZAMH Zabidi-Hussin}

It has long been assumed that children are 'mini adults" and whatever 'things' relevant to adults, could be scaled down to children. Unfortunately these assumptions include critical issues such research findings, medications and to some extent, to the use of medical devices and have great implications that can be detrimental to children.For a long time, clinical researches involving children have often been seen as something unethical; as they are classified as vulnerable groups and must be protected. As a result of all these assumptions and obstacles, consideration their complex developmental stages, maturity of the body system and psychological developments. The need for developmentally appropriate outcome measures for children of different ages, the complexities of parental involvement and family decision-making also add to another dimension and must be taken into account in recherch procedures and settings involving children.

This article attempts to justify why clinical researches involving children are needed and also suggest how these researches could be done in an ethical manner. Children undergo rapid developmental changes, marked by combination of physical and emotional maturation, and enhanced by their nutritional intake. These changes produce great net impact on their abilities to metabolize drugs as the specific drug receptors are also affected by the maturity of the body system.Drug receptor development in children require a very complex study as the various drug receptors may develop with advancing age and represent a big challenge to drug discovery.

These pharmacodynamics changes have not been studied well and clearly have important implications on children. Phenobarbital as an example may produce sedation in adults but paradoxically cause excitation and hyperactivity in children, thought to be related to central nervous system receptors immaturity.

Clearly extrapolating findings from researches done in the adult population in terms of drug development will simply not work under these circumstances.

Some diseases are also unique to children, making interventions and treatment unique and must be clearly targeted. Conditions such as prematurity and its sequelae can occur only in this age group. Similarly, certain genetic conditions such as phenylketonuria (PKU) or other metabolic disorders will, if untreated, lead to severe disabilities or even death in childhood. Hence all issues pertaining to updated management of these conditions must be continuously updated through researches involving these children.

Cancers in children have unique pathophysiology, severities and follow certain course, which are often determined by a number of non-quantifiable factors such as family environment and social support. Their response $\mathrm{s}$ to treatment may thus differ fromthe treatment course in adults. Hence safe and effective treatment for adults may not necessarily be applicable to children.Similarly when the treatment of these malignancies involves radiation therapy, the tissue irradiation can disrupt normal tissue development in children, leading to possible consequences such as growth and development failures. Such failures may not affect the adult population.

Medical devices that are placed in children are also closely linked to their developmental stages. Devices such as intraocular lens replacement after cataract surgery have been the mainstay of treatment of cataract in the adult and children, but developmental issues in children present unique challenges to this age group.As an example the greater elasticity of the anterior capsules, changes the refractive state of the children's eyes.Researches are therefore needed as the findings are critical to guide medical practitioners' when recommending such devices to children.

Correspondence to: Prof Dr. ZAMH Zabidi-Hussin, Professor of Paediatrics and Director of Postgraduate Studies, Research and Innovation, MAHSA University, Selangor, Malaysia. 
The American Academy of Pediatrics (AAP) noted that the shortage of paediatric research creates an ethical dilemma for physicians, who "must frequently either not treat children with potentially beneficial medications or treat them with medications based on adult studies or anecdotal empirical experience in children" It is somewhat disappointing to discover that approximately 80 percent of medications listed in the Physician's Desk Reference had labels that provided no prescribing information for childrenThe report also found that 62 percent lacked labeling information for paediatric use at the time that they were initially approved for marketing It is extremely disturbing that when drug labels lack paediatric prescribing information, physicians can still legally prescribe drugs for children on an "off-label" basis. In one of a European study, it was discovered that at least one-third of hospitalized children and up to 90 percent of neonates in intensive care receive such prescriptions.

The way forward

Obstacles relate to undertaking researches in children are often attributed to assigning them as vulnerable group and need to be protected. An Institutional Review Board (IRB) specific to address the research issues in the paediatric population is needed. This IRB should have its own advisor from the fraternity of scientists who are familiar with the need to do these researches and also with the sound knowledge of paediatric population and their families. There is always a possibility that children who are involved in researches are not able to willingly give consents but became involved in the researches through assents, given by their care givers. As such they may not have full understanding of the said research and may display signs of distress. It is important that such signs are monitored, preferably by child psychologists who may be a member of the IRB or part of the Ethics Advisor's team and also play the role of giving emotional support to these children throughout the lifetime of the project. Similarly a paediatric nurse who is fully experienced and has practical knowledge of babies and children should be involved in the ethics advisor team too. The Royal College of Paediatrics and Child Health of the UK, in its Ethics Advisory Committee Guidelines categorized research procedures into minimum, low and high risk, where minimum risk is defined as researches involving questioning, observing, and measuring children or those that involve processing secondary data. High risks procedures involve invasive techniques, which must only done at the same time as the child is undergoing treatment, operative or invasive diagnostic procedure. A non-therapeutic reason for blood taking is thus completely unacceptable. Assents in researches involving children should also be revised as the children reach maturity and their decisions on continued participation or otherwise should be respected.

\section{Conclusion}

In as much as researches in children are necessary for a number of reasons, specific considerations should be taken into account to avoid any undue distress on these children and their families

\section{References:}

1. Ethical Conduct of Clinical Research Involving Children (2004).Institute of Medicine 2004. Washington, DC: The National Academies Press.

2. https://doi.org/10.17226/10958. https://doi.org/10.17226/10958

3. Jean-Marie Nicolas et al Oral drug absorption in pediatrics: the intestinal wall, its developmental changes and current tools for predictions. Biopharm. Drug Dispos. 38: 209-230 (2017)

\section{https://doi.org/10.1002/bdd.2052}

4. Mohammed Al Shamrani, Shahira Al Turkmani, Pediatric Ophthalmology Update : Update of intraocular lens implantation in children

5. Saudi Journal of Ophthalmology (2012) 26, 271-275 https://doi.org/10.1016/j.sjopt.2012.05.005

6. Royal College of Paediatrics and Child Health: Ethics Advisory Committee 